Characterization of the low-frequency noise in high-temperature radio frequency superconducting quantum interference devices cooled in static magnetic fields

J. Borgmann, P. David, R. Otto, J. Schubert, and A. I. Braginski

Citation: Appl. Phys. Lett. 74, 1021 (1999);

View online: https://doi.org/10.1063/1.123442

View Table of Contents: http://aip.scitation.org/toc/apl/74/7

Published by the American Institute of Physics

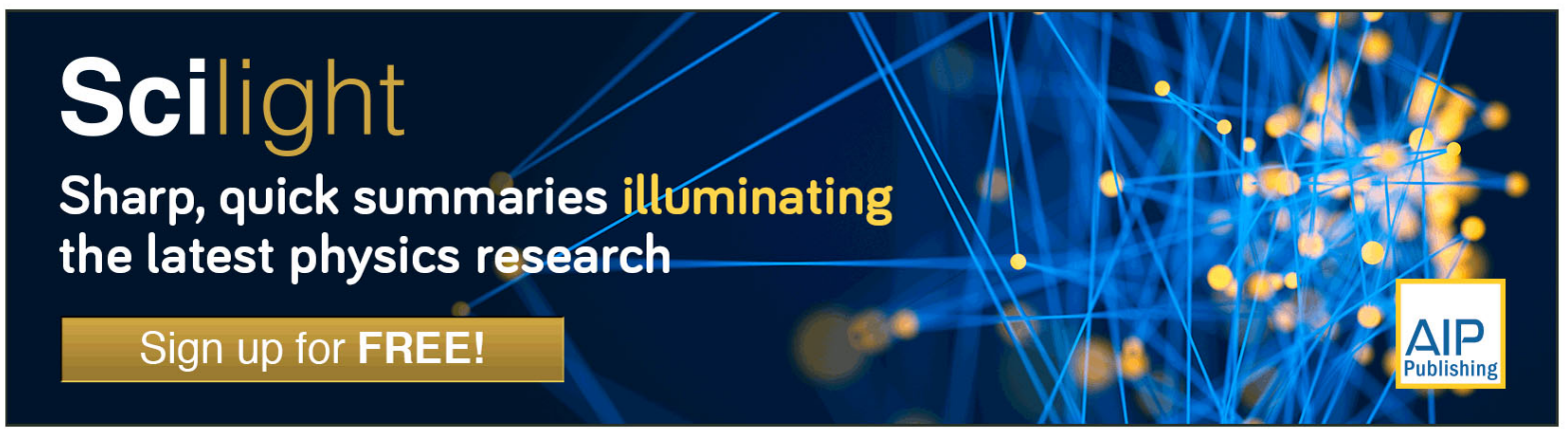




\title{
Characterization of the low-frequency noise in high-temperature radio frequency superconducting quantum interference devices cooled in static magnetic fields
}

\author{
J. Borgmann and P. David \\ Institut für Strahlen- und Kernphysik der Universität Bonn, D-53117 Bonn, Germany
}

R. Otto, J. Schubert, and A. I. Braginski

Institut für Schicht- und Ionentechnik, Forschungszentrum Jülich GmbH (FZJ), D-52425 Jülich, Germany

(Received 8 June 1998; accepted for publication 1 December 1998)

\begin{abstract}
We have performed a study of the low-frequency noise in high-temperature radio frequency (rf) superconducting quantum interference devices (SQUIDs) cooled in static magnetic fields. At $77 \mathrm{~K}$, the noise of different thin-film $\mathrm{YB}_{2} \mathrm{C}_{3} \mathrm{O}_{7-x}$ washer rf SQUIDs was measured in static magnetic fields up to $55 \mu \mathrm{T}$. The SQUID inductances ranged from 150 to $300 \mathrm{pH}$. The measured low-frequency flux noise of the SQUIDs $S_{\Phi}^{1 / 2}$ at $1 \mathrm{~Hz}$ was between 70 and $350 \mu \Phi_{0} /{ }_{\sqrt{ }} \mathrm{Hz}$, and in terms of magnetic field noise $S_{B}^{1 / 2}$ between 120 and $1100 \mathrm{fT} / \sqrt{ } \mathrm{Hz}$. The low-frequency noise of all devices at $1 \mathrm{~Hz}$ showed no significant increase for all applied static fields. (C) 1999 American Institute of Physics. [S0003-6951(99)01306-6]
\end{abstract}

In recent years, several types of low-noise hightemperature superconductor (HTS) superconducting quantum interference device (SQUIDs) were fabricated. Their application for biomagnetic measurements was demonstrated in magnetically shielded environments. ${ }^{1-5}$ Most of these devices showed additional low-frequency noise. For dc SQUIDs the fluctuation of the junctions critical current $I_{c}$ and the motion of flux vortices have been identified as $1 / f$ noise sources. ${ }^{6}$ In $\mathrm{rf}$ SQUIDs only noise of the vortex motion should be observable.

For widespread use of SQUIDs, the capability of operating in the earth's magnetic field $(\sim 50 \mu \mathrm{T})$ is required. Therefore, several studies on the white and low-frequency noise performance in static magnetic fields were performed. $^{7-9}$ The devices investigated also showed changes in the white noise level, which were dependent on the junction width and the current distribution over the junction. ${ }^{6,8}$

Until now, the low-frequency $1 / f$-type noise in static magnetic fields was systematically investigated only in HTS dc SQUIDs. Typically, there was a strong linear increase of $1 / f$ noise with increasing magnetic field intensity in which the SQUID was cooled from above the critical temperature $T_{c} \cdot{ }^{7-10}$ A sufficient reduction of the width of dc SQUID thin-film structures made it possible to operate them with minimal increase of noise up to values of field exceeding that of the earth. ${ }^{10,12}$ While no systematic investigation of single rf SQUIDs magnetometers existed until now, we note that highly balanced second-order electronic gradiometers based on HTS rf SQUIDs showed relative small low-frequency noise increases when operated in the earth's magnetic field. ${ }^{13}$

In the present work, a study of the low-frequency noise performance of different types of rf SQUID magnetometers has been performed. Special measuring methods have to be used due to the fact that a static magnetic field of typical earth field intensity $(55 \mu \mathrm{T})$ needed for the noise characterization must have an instability not exceeding $10^{-10} .^{7} \mathrm{We}$ have chosen a technique, which allows us only an indirect measurement of the magnetometer noise, but excludes adequately the static field fluctuations originating from the cur- rent supply of the normal magnetizing coil. A setup with two magnetometers was chosen to measure in a differential mode, in order to suppress the common-mode fluctuations of the generated static field. Figure 1 shows schematically the arrangement with the magnetizing solenoid inside a fourlayer set of cylindrical mumetal shields closed at the bottom. The 50-cm-long solenoid, $21 \mathrm{~cm}$ in diameter, could produce a static magnetic field up to $55 \mu \mathrm{T}$. The field of the coil was measured along its axis with a fluxgate sensor. Due to the large coil volume, the magnetic field in the center of the coil varied only by about $1 \%$ over the axial length of $20 \mathrm{~cm}$. In

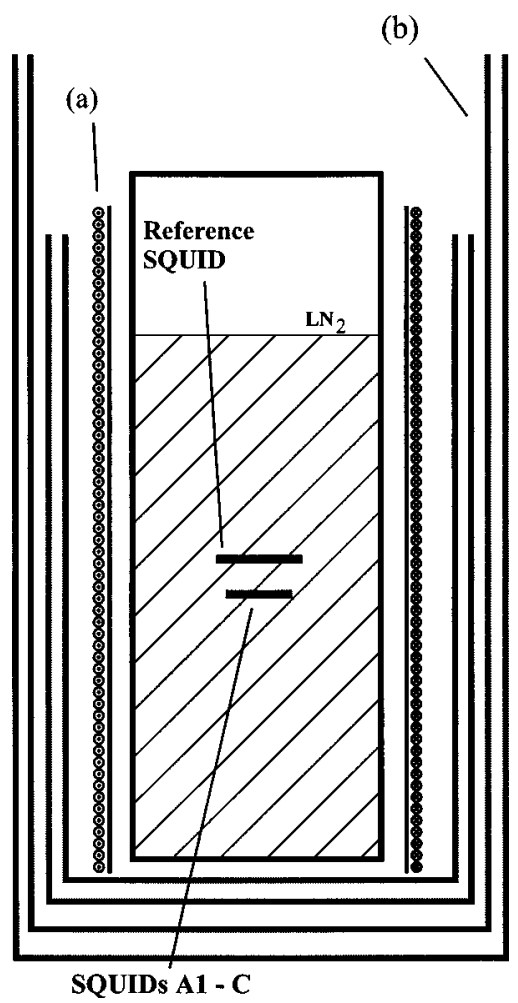

FIG. 1. Schematic transverse section drawing of the setup for the measurement of low-frequency noise in static fields: (a) coil for the generation of the static field, (b) four layer mumetal shielding. 
the center of the coil, a set of two parallel magnetometers was placed within an axial distance of $1.7 \mathrm{~cm}$ such that their planes were oriented perpendicular to the static magnetic field. The mechanical misorientation of the magnetometer planes for all setups was less than $0.3^{\circ}$. The magnetometers were operated in the flux locked loop (FFL) configuration. The outputs of two FFLs were used to form an electronic gradiometer. ${ }^{13}$ The gradiometer was electronically tuned not for ideal gradiometric output, but for maximum commonmode rejection (CMR) of the field produced by the coil. Typical CMR values were better than 5000, which would be enough to suppress the spectral noise [Figs. 2(b) and 2(c)] generated in the coil to a value of $2 \mathrm{fT} / \sqrt{ } \mathrm{Hz}$ at $1 \mathrm{~Hz}$ for fields of $55 \mu \mathrm{T}$. This value is much lower than the white noise level of our HTS-rf-SQUID magnetometers and can be neglected.

The tank circuit frequency of the rf SQUIDs ranged between 170 and $270 \mathrm{MHz}$. Reference 13 gives a detailed description of the electronic and high-frequency coupling scheme used in the setup. For the measurement, the two SQUIDs were mounted and operated in a low noise fiberglass Dewar filled with $\mathrm{LN}_{2}$, as described in Ref. 13. Five different types of magnetometers were examined: three single washer SQUIDs and two washer SQUIDs combined with either a bulk flux focusser or a thin-film, single-layer flux transformer. The SQUID washers were patterned from superconducting films of typical $200 \mathrm{~nm}$ thickness, grown by pulsed laser deposition on $\mathrm{LaAlO}_{3}$ substrates. ${ }^{14}$ The singlelayer thin-film flux transformers were magnetron sputtered on $\mathrm{CeO}_{2}$-buffered sapphire. ${ }^{15}$ The SQUID inductances ranged from 150 to $300 \mathrm{pH} .{ }^{14}$ In flip-chip configurations, only the $300 \mathrm{pH}$ square washer SQUIDs were used. Table I gives an overview of the parameters of the different magnetometers including the reference magnetometers (RMs). The square washer $300 \mathrm{pH}$ SQUID (Ref. 1) was used as a reference magnetometer for the magnetometers $A 1, B$, and $C$ studied. For flip-chip magnetometers $A 2$ and $A 3$, the reference magnetometer (Ref. 2) was used, combining the washer SQUID with a flip-chip single-layer thin-film flux transformer.

The performance of the setup of Fig. 1 is illustrated in Fig. 2, which shows noise spectra measured with a washer SQUID combined with a thin-film single layer transformer without applying any field (a), and after cooling from above $T_{c}$ in fields of 20 and $55 \mu \mathrm{T}$ [(b) and (c)]. After each field application and measurement, the SQUID was degaussed by heating above $T_{c}$. Before the field was applied, the $1 / f$ noise was below the white noise floor down to $0.6 \mathrm{~Hz}$. In the

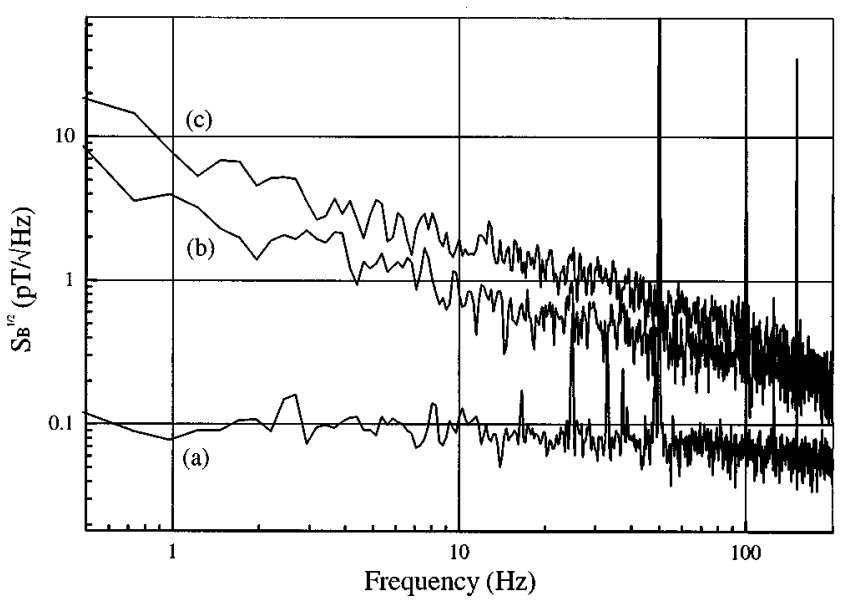

FIG. 2. Magnetic noise spectra $S_{B}^{1 / 2}$ : (a) without applied field, (b) in $20 \mu \mathrm{T}$, and (c) in $55 \mu \mathrm{T}$, including the fluctuations of the coil power supply.

presence of field, the increase of noise observed at $1 \mathrm{~Hz}$ reached 2 orders of magnitude in $55 \mu \mathrm{T}$. This increase is caused by the current noise of the coil supply, as the lowfrequency noise level in the gradiometric measurements was much lower.

For measurements in the gradiometric configuration, i.e., excluding the common mode, the SQUID to be tested and the RM were first cooled in zero magnetic field. Subsequently, the gradiometer was electronically balanced in a weak ac field generated by the field coil to attain the maximum CMR. Due to the different effective areas of the RM and of the magnetometer under test, their output signal amplitudes were different. This led to equivalent flux noise levels at the differential gradiometer output, which sometimes were much higher than that of the RM flux noise level.

After measuring the spectral flux noise of each magnetometer and of the balanced gradiometer in zero field, the static field was increased to $20 \mu \mathrm{T}$ while the SQUIDs were cold and the flux noise was measured at the gradiometric output. Subsequently, the SQUIDs were heated above the critical temperature $T_{c}$, cooled to $77 \mathrm{~K}$, and the flux noise at the gradiometric output was measured again. Then a field of $40 \mu \mathrm{T}$ was applied, and the spectral noise was measured again. This procedure was repeated in an analogous way for $55 \mu \mathrm{T}$. Figure 3 shows the equivalent flux noise spectra of the electronic gradiometer in zero field and at 20 and $40 \mu \mathrm{T}$ without raising the temperature above the critical temperature. In $20 \mu \mathrm{T}$ applied at $77 \mathrm{~K}$, the noise at $1 \mathrm{~Hz}$ increased by a factor greater than 10 .

In contrast, when a field was applied above $T_{c}$ and the

TABLE I. Data and performance of the rf SQUID magnetometers.

\begin{tabular}{|c|c|c|c|c|c|c|c|}
\hline $\begin{array}{l}\text { SQUID: } \\
\text { Washer-type }\end{array}$ & $\begin{array}{l}\text { Ref. } 1 \\
\text { Square }\end{array}$ & $\begin{array}{l}\text { Ref. } 2 \\
\text { Square }\end{array}$ & $\begin{array}{c}A 1 \\
\text { Square }\end{array}$ & $\begin{array}{c}A 2 \\
\text { Square }\end{array}$ & $\begin{array}{c}A 3 \\
\text { Square }\end{array}$ & $\begin{array}{c}B \\
\text { Circular }\end{array}$ & $\begin{array}{c}C \\
\text { Circular }\end{array}$ \\
\hline $\begin{array}{l}\text { Single layer } \\
\text { flux transformer }\end{array}$ & - & $\begin{array}{l}\text { Thin film } \\
23 \mathrm{~mm} \varnothing\end{array}$ & - & $\begin{array}{l}\text { Thin film } \\
23 \mathrm{~mm} \varnothing\end{array}$ & $\begin{array}{c}\text { Bulk } \\
25 \mathrm{~mm} \varnothing\end{array}$ & - & - \\
\hline SQUID hole area $\left(\mu \mathrm{m}^{2}\right)$ & $200 \times 200$ & $200 \times 200$ & $200 \times 200$ & $200 \times 200$ & $200 \times 200$ & $100 \times 100$ & $20 \times 500$ \\
\hline Washer area $\left(\mathrm{mm}^{2}\right)$ & $8 \times 8$ & $8 \times 8$ & $8 \times 8$ & $8 \times 8$ & $8 \times 8$ & $3.5 \varnothing$ & $3.5 \varnothing$ \\
\hline SQUID inductance $(p \mathrm{H})$ & 300 & 300 & 300 & 300 & 300 & 150 & 240 \\
\hline White flux noise $\left(\mu \Phi_{0} / \sqrt{\mathrm{Hz}}\right)$ & 70 & 70 & 70 & 70 & 70 & 35 & 50 \\
\hline$\delta B / \delta \Phi\left(\mathrm{nT} / \Phi_{0}\right)$ & 1.93 & 0.75 & 1.95 & 0.74 & 0.63 & 10 & 11.1 \\
\hline White field noise $\left(\mathrm{fT} /{ }_{\sqrt{ }} \mathrm{Hz}\right)$ & 135 & 53 & 137 & 52 & 44 & 350 & 555 \\
\hline
\end{tabular}




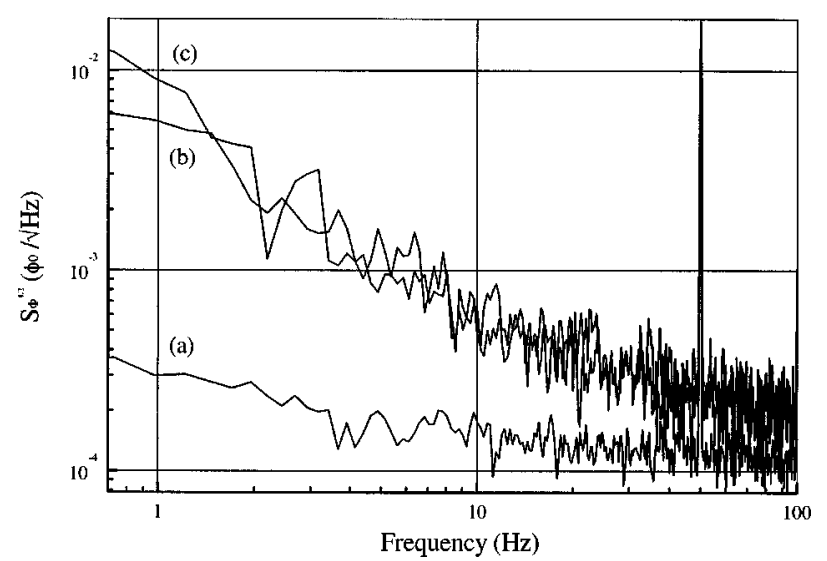

FIG. 3. Flux noise spectra $S_{\phi}^{1 / 2}$ of the electronic gradiometer in zero field (a), after changing the static field in steps of $20 \mu \mathrm{T}$ from 0 to $20 \mu \mathrm{T}$ (b) and from 20 to $40 \mu \mathrm{T}$ (c), with the SQUIDs kept at a constant temperature of 77 K.

SQUID was cooled, no significant increases of equivalent flux noise could be observed at the gradiometer output. To obtain the individual flux noise of a given magnetometer $S_{\Phi M}^{1 / 2}$, the noise of the reference has been subtracted as follows:

$$
S_{\Phi M}^{1 / 2}=\left(S_{\Phi G}-\alpha S_{\Phi R}\right)^{1 / 2},
$$

where $S_{\Phi G}^{1 / 2}$ is the gradiometer noise, $S_{\Phi R}^{1 / 2}$ is the reference noise, and $\alpha$ is the ratio of the effective area of the RM and the magnetometer. Because the noise of RM in static fields was not known, its noise measured in zero static field was subtracted from the gradiometric signal for all fields, thus giving only an upper limit of noise originating from the magnetometer under test. For magnetometers having small effective area in comparison to RM, the obtained $S_{\Phi M}^{1 / 2}$ values are much lower than the gradiometric equivalent flux noise $S_{\Phi G}^{1 / 2}$. Figure 4 summarizes the resulting flux noise data at 1 $\mathrm{Hz}$ for all tested magnetometers cooled down in 0, 20, 40, and $55 \mu \mathrm{T}$. The minimal low frequency flux noise of about $70 \mu \Phi_{0} / \sqrt{ } \mathrm{Hz}$ at $1 \mathrm{~Hz}$ is achieved for the slot hole washer SQUID $C$. The lowest $1 / f$ field noise $120 f \mathrm{~T} / \sqrt{ } \mathrm{Hz}$ was achieved by SQUID A3 due to the high effective area.

The measurement of the flux noise at $1 \mathrm{~Hz}$ in static magnetic fields shows different results from those reported in the literature. The flux noise at $1 \mathrm{~Hz}$ has no significant increase in static magnetic fields for the used large washer $\mathrm{rf}$

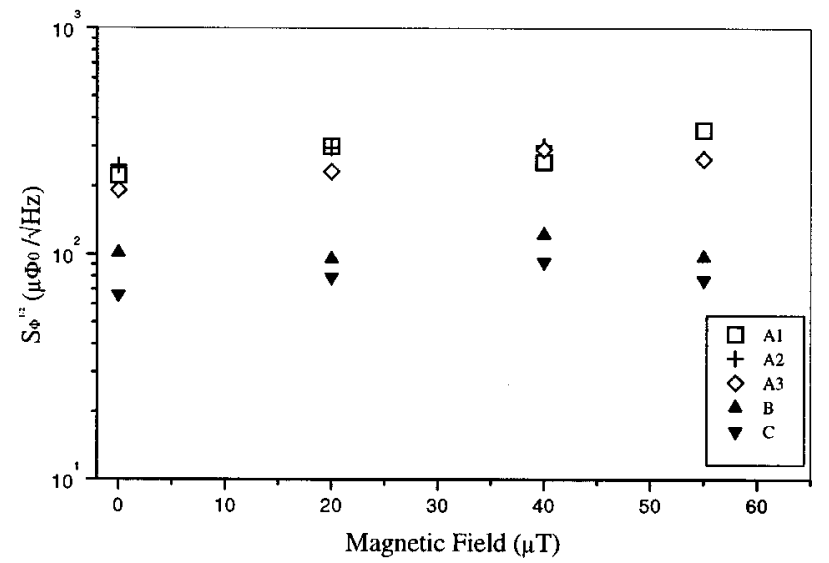

FIG. 4. Flux noise $S_{\phi}^{1 / 2}$ at $1 \mathrm{~Hz}$ for the SQUID magnetometers vs static fields applied when cooling the setup from above $T_{c}$.
SQUIDs. The threshold field $B_{0}$ of $\pi \Phi_{0} / 4 w^{2}$ for dc SQUIDs examined in Ref. 11 is below $1 \mathrm{nT}$ for our smallest washers. Therefore, the low-frequency noise power should increase even for low static fields and scale linearly with their intensity. The rf SQUIDs examined in this work have three to six times higher inductances than the HTS dc SQUIDs of Ref. 11 and higher white flux noise levels. In direct comparison the lowest measured flux noise at $1 \mathrm{~Hz}$ of $70 \mu \Phi_{0} / \sqrt{ } \mathrm{Hz}$ in a static field of $55 \mu \mathrm{T}$ for SQUID $C$ is lower than the flux noise levels reported in the literature. ${ }^{7,11}$

One hypothetical explanation for the observed independence of low-frequency noise level upon the applied static field may be the rf field applied to the sensor. Schöne et al. reported that the application of strong if fields above 100 $\mathrm{MHz}$ to HTS-SQUID sensors leads to a reduction of the low-frequency noise. ${ }^{16}$ In our case, due to the fact that the tank circuit has a good coupling to the washer SQUID, the applied rf currents in the thin film might have been sufficiently high to excite and displace the vortices to local potential minima, where they could be effectively pinned. The excitation level could have been sufficient when cooling the washers from above $T_{c}$, but too low to be effective when static fields were applied to a cold washer. Another possibility is that the noise of our HTS thin films is much lower than in the literature, despite the low $T_{c}$ of $87 \mathrm{~K}$ for our films. ${ }^{7,11,12}$ As the measured flux noise scales with the noise of the thin film the contribution might be below the white noise level of our sensors and the level in earlier publications. ${ }^{7,11,12}$ To verify the reason for the constant noise level at low frequencies, further studies should be conducted using SQUIDs having lower white flux noise levels than those tested in this work.

${ }^{1}$ Y. Zhang, M. Mück, K. Herrmann, J. Schubert, W. Zander, A. I. Braginski, and C. Heiden, IEEE Trans. Appl. Supercond. AS-3, 2465 (1993).

${ }^{2}$ D. Koelle, A. H. Miklich, F. Ludwig, E. Dantsker, D. T. Nemeth, and J. Clarke, Appl. Phys. Lett. 63, 2271 (1993).

${ }^{3}$ F. Ludwig, E. Dantsker, R. Kleiner, D. Koelle, J. Clarke, S. Knappe, D. Drung, H. Koch, N. McN. Alford, and T. W. Button, Appl. Phys. Lett. 66, 1418 (1995).

${ }^{4}$ Y. Q. Shen, Z. J. Sun, R. Kromann, T. Holst, P. Vase, and T. Freloft, Appl. Phys. Lett. 67, 2081 (1995).

${ }^{5}$ J. Beyer, D. Drung, F. Ludwig, T. Minotani, and K. Enpuku, Appl. Phys. Lett. 72, 203 (1998).

${ }^{6}$ A. H. Miklich, J. Clarke, M. S. Colclough, and K. Char, Appl. Phys. Lett. 60, 1899 (1992).

${ }^{7}$ A. H. Miklich et al. Appl. Phys. Lett. 64, 3494 (1994).

${ }^{8}$ V. N. Glyantsev, Y. Tavrin, W. Zander, J. Schubert, and M. Siegel, Semicond. Sci. Technol. 9, A105 (1996).

${ }^{9}$ J. M. Schmidt, L. P. Lee, A. Matlashov, M. Teepe, V. Vinetsky, R. Cantor, and M. S. Dilorio, Proceedings of the Biomagnetic Conference 96, Santa Fe, New Mexico, February 16-21, 1996.

${ }^{10}$ F. P. Milliken, S. L. Brown, and R. H. Koch, Appl. Phys. Lett. 71, 1857 (1997).

${ }^{11}$ E. Dantsker, S. Tanaka, P. Å. Nilsson, R. Kleiner, and J. Clarke, Appl. Phys. Lett. 69, 4099 (1996).

${ }^{12}$ E. Dantsker, S. Tanaka, and J. Clarke, Appl. Phys. Lett. 70, 2037 (1997).

${ }^{13}$ J. Borgmann, G. Ockenfuss, R. Otto, J. Schubert, W. Zander, A. I. Braginski, and P. David, Rev. Sci. Instrum. 68, 2730 (1997).

${ }^{14}$ Y. Zhang, W. Zander, J. Schubert, F. Ruders, H. Soltner, M. Banzet, N. Wolters, X. H. Zeng, and A. I. Braginski, Appl. Phys. Lett.71, 704 (1997).

${ }^{15}$ G. Ockenfuss, J. Borgmann, M. Reese, and R. Wördenweber, IEEE Trans. Appl. Supercond. 7, 3698 (1997).

${ }^{16}$ S. Schöne, M. Mück, and C. Heiden, Appl. Phys. Lett. 68, 859 (1996). 